Bull. Austral. Math. Soc.

Vol. 37 (1988) [121-130]

\title{
A HIGHER ORDER APPROXIMATION TECHNIQUE FOR RESTRICTED LINEAR LEAST-SQUARES PROBLEMS
}

\author{
Heinz W. Engl and C. W. Groetsch
}

\begin{abstract}
An essential limitation for the method of weighting for equality constrained linear leastsquares problems is the sub-optimality of the attainable convergence rate. In this paper, we propose a method, related to the method of iterated Tikhonov regularisation, that (under suitable conditions) gives rise to convergence rates which are arbitrarily near the optimal rate. As a by-product, we develop the theory of iterated Tikhonov regularisation for equations with unbounded linear operators.
\end{abstract}

\section{INTRODUC'TION}

The "method of weighting" is concerned with approximating the vector $\hat{x}$ which is a least-squares solution of

$$
K x=g
$$

and which, among all least-squares solutions of (1), satisfies also

$$
L x=f
$$

in the least-squares sense. Here $K: H_{1} \rightarrow H_{2}$ and $L: H_{1} \rightarrow H_{3}$ are linear operators between the Hilbert spaces $H_{1}, H_{2}, H_{3}$. $K$ (but not $L$ ) will always be assumed to be bounded. Throughout the paper we will assume that the product operator $(K, L): H_{1} \rightarrow H_{2} \times H_{3}$ has a trivial nullspace and a closed range. For a discussion of these conditions see [2]. The "restricted least-squares solution" $\hat{x}$ can be defined more formally by

$$
\|K \hat{x}-g\|=\inf \left\{\|K x-g\| \mid x \in H_{1}\right\}
$$

and

$$
\|L \hat{x}-f\|=\inf \{\|L \bar{x}-f\| \mid \bar{x} \operatorname{solves}(3)\}
$$

Received 7 April 1987

Supported by the Austrian Fonds zur Förderung der wissenschaftlichen Forschung (H.W.E., project S32/03) and the NSF (C.W.G., grant INT-8510037)

Copyright Clearance Centre, Inc. Serial-fee code: 0004-9729/88 \$A2.00+0.00. 
The theory of, and approximation methods for restricted least-squares solutions have been developed in detail in [2] (see [4], [11]) independently from [17], which also contains related results. Restricted least-squares solutions in this sense have already been discussed in [18, p234]. As Nashed has informed us, some of the theory and approximation results for restricted least-squares solutions can be derived from the results of his joint paper with Lee, to be published (see the abstract [14] and [15]). In the special case when $L=I$ and $f=0, \hat{x}=K^{\dagger} g$, where $K^{\dagger}$ is the Moore-Penrose generalised inverse of $K$ (see [18]). Another special case that has received attention is the case when $f=0$ and $L$ is a differential operator $([\mathbf{1 6}],[20],[5],[7])$; for this "regularisation with a differential operator" also the case $f \neq 0$ could be of interest. For other problems that fit into the framework of "restricted least-squares problems" see $[2],[11]$.

If $f \in D\left(L^{\dagger}\right)$, then one can decompose the restricted least-squares solution as

$$
\hat{x}=\mathrm{K}^{\dagger} g+P L^{\dagger} f
$$

where $\mathrm{K}$ denotes the operator $K$ acting on $H_{1}$, endowed with the inner product

$$
[x, y]:=\langle K x, K y\rangle+\langle L x, L y\rangle
$$

(which is again a Hilbert space), and $P$ is the $[\cdot, \cdot]$-orthogonal projector of $H_{1}$ onto $N(K)$ (see [4],[11]). Note that the norm induced by $[\cdot, \cdot]$ is equivalent to the original norm on $H_{1}$ and played a crucial role in the special setting of [16]. Hence, we will denote both norms on $H_{1}$ by $\|\cdot\|$.

Since $P=I-\mathrm{K}^{\dagger} \mathrm{K}$, we may write (5) as

$$
\hat{x}=L^{\dagger} f+\mathrm{K}^{\dagger}\left(g-\mathrm{K} L^{\dagger} f\right)
$$

The method of weighting for approximating $\hat{x}$ results in approximations $x_{\epsilon}$, defined as minimisers of the functional $u \rightarrow\|K u-g\|^{2}+\epsilon\|L u-f\|^{2}$. It follows from the representations of $x_{\epsilon}$ given in [11] that $x_{\epsilon}$ can be written as

$$
x_{\epsilon}=L^{\dagger} f_{+}(1+\alpha)\left[\left(\alpha I+\mathrm{K}^{\star} \mathrm{K}\right)^{-1} \mathrm{~K}^{\star}\right]\left(g-\mathrm{K} L^{\dagger} f\right) \text {, }
$$

where $\epsilon$ and $\alpha$ are related by $\epsilon=\frac{\alpha}{1+\alpha}$ and $\mathrm{K}^{\star}$ is the adjoint of $\mathrm{K}$, if $H_{1}$ is equipped with the inner product $[\cdot, \cdot]$. Comparing (7) and (8), one can interpret the method of weighting as an application of ordinary Tikhonov regularisation for approximating $\mathrm{K}^{\dagger}$ in (7). This leads to the idea of designing a method with a higher rate of convergence by replacing Tikhonov regularisation in (8) by a method that converges faster, for example, iterated Tikhonov regularisation (of order $n$ ). Simultaneously, we will also approximate 
$L^{\dagger} f$ in (7) by iterated Tikhonov regularisation (of order $n$ ). This leads to the following approximation $y_{\alpha, n}$ for $L^{\dagger} f$ :

$$
y_{\alpha, 0}:=0, \quad\left(L^{\star} L+\alpha I\right) y_{\alpha, j}=L^{\star} f+\alpha y_{\alpha, j-1} \quad(j=1, \ldots, n)
$$

For approximating the second term in (7) alone (without simultaneously approximating $\left.L^{\dagger} f\right)$, we would use $w_{\alpha, n}$ defined by

$$
w_{\alpha, 0}:=0, \quad\left(\mathrm{~K}^{\star} \mathrm{K}+\alpha I\right) w_{\alpha, j}=\mathrm{K}^{\star}\left(g-\mathrm{K} L^{\dagger} f\right)+\alpha y_{\alpha, j-1} \quad(j=1, \ldots, n) .
$$

However, since we also want to approximate $L^{\dagger} f$ simultaneously, we replace $w_{\alpha, n}$ by $z_{\alpha, n}$ defined by

$$
z_{\alpha, 0}:=0, \quad\left(\mathrm{~K}^{\star} \mathrm{K}+\alpha I\right) z_{\alpha, j}=\mathrm{K}^{\star}\left(g-\mathrm{K} y_{\alpha, n}\right)+\alpha z_{\alpha, j-1} \quad(j=1, \ldots, n)
$$

and use

$$
x_{\alpha, n}:=y_{\alpha, n}+z_{\alpha, n}
$$

as our approximation for $\hat{x}$. In view of (8), one might be tempted to multiply $z_{\alpha, n}$ in (12) by $(1+\alpha)$; however, this would slow down the rate of convergence to at most $O(\alpha)$.

We now formulate the method in a way involving only the original operators $L$ and $K$ (and not $\mathrm{K}$ ) and their adjoints (with respect to the original inner products):

Algorithm. Let $n \in \mathrm{N}, \alpha>0$ and define $y_{\alpha, n}$ by (9). Let $z_{\alpha, n}$ be defined by $z_{\alpha, 0}:=0$,

$$
\left[(1+\alpha) K^{\star} K+\alpha L^{\star} L\right] z_{\alpha, j}=K^{\star} g-K^{\star} K y_{\alpha, n}+\alpha\left(L^{\star} L+K^{\star} K\right) z_{\alpha, j-1}(j=1, \ldots, n)
$$

and define $x_{\alpha, n}$ by (12).

Since $K^{\star}=\left(L^{\star} L+K^{\star} K\right)^{-1} K^{\star},(11)$ and (13) are equivalent.

Note that, for the moment, $(9)$ is meaningful only for bounded $L$. What we mean by (9) for unbounded $L$ will be made clear in Section 3 . For the method of weighting it is known $([2],[11])$ that if the data $g$ and $f$ are subject to perturbations of magnitude $\delta$ (and the problem is ill-posed, that is, $R(K)$ is non-closed), then the best possible asymptotic rate of convergence for the approximants using the perturbed data is $O\left(\delta^{\frac{2}{3}}\right)$, no matter what choice of $\alpha=\alpha(\delta)$ one takes and what a-priori assumptions one makes. We will show that our algorithm can have the convergence rate $O\left(\delta^{\nu}\right)$ for any $\nu<1$ under suitable conditions. 


\section{THE CASE OF BOUNDED OPERATORS}

We consider first the case in which both the operators $K$ and $L$ are bounded. The following facts are found in [13]: If $L^{\dagger} f \in R\left(\left(L^{\star} L\right)^{n}\right)$, then :

$$
\left\|y_{\alpha, n}-L^{\dagger} f\right\|=O\left(\alpha^{n}\right)
$$

if only a perturbed version $f_{\delta}$ of $f$ satisfying $\left\|f-f_{\delta}\right\| \leqslant \delta$ is available, then

$$
\left\|y_{\alpha, n}^{\delta}-y_{\alpha, n}\right\|=O\left(\frac{\delta}{\sqrt{\alpha}}\right)
$$

where $y_{\alpha, n}^{\delta}$ is defined by (9) with $f$ replaced by $f_{\delta}$. Then similarly, if $g-\mathrm{K} L^{\dagger} f \in$ $R\left(\left(\mathrm{~K}^{\star} \mathrm{K}\right)^{n}\right)$, the approximations $w_{\alpha, n}$ defined by $(10)$ satisfy

$$
\left\|w_{\alpha, n}-K^{\dagger}\left(g-K L^{\dagger} f\right)\right\|=O\left(\alpha^{n}\right) .
$$

Now we show by induction that

$$
\left\|w_{\alpha, n}-z_{\alpha, n}\right\|=O\left(\alpha^{n}\right)
$$

where $z_{\alpha, n}$ is defined by (11): For all $j \in(1, \ldots, n)$,

$$
w_{\alpha, j}-z_{\alpha, j}=\alpha\left(\mathrm{K}^{\star} \mathrm{K}+\alpha I\right)^{-1}\left(w_{\alpha, j-1}-z_{\alpha, j-1}\right)-\left(\mathrm{K}^{\star} \mathrm{K}+\alpha I\right)^{-1} \mathrm{~K}^{\star} \mathrm{K}\left(L^{\dagger} f-y_{\alpha, n}\right) .
$$

Since

$$
\left\|\left(\mathrm{K}^{\star} \mathrm{K}+\alpha I\right)^{-1} \mathrm{~K}^{\star} \mathrm{K}\right\| \leqslant 1
$$

as can be seen using spectral theory, and since $w_{\alpha, 0}=z_{\alpha, 0}=0$, it follows from (14) that $\left\|w_{\alpha, 1}-z_{\alpha, 1}\right\|=O\left(\alpha^{n}\right)$. Now assume that for some $j \geqslant 2,\left\|w_{\alpha, j-1}-z_{\alpha, j-1}\right\|=O\left(\alpha^{n}\right)$. Then it follows from the formula for $w_{\alpha, j}-z_{\alpha, j}$, together with (14), (18) and the estimate

$$
\left\|\left(\mathrm{K}^{\star} \mathrm{K}+\alpha I\right)^{-1}\right\|=O\left(\frac{1}{\alpha}\right)
$$

that $\left\|w_{\alpha, j}-z_{\alpha, j}\right\|=\alpha \cdot O\left(\frac{1}{\alpha}\right) \cdot O\left(\alpha^{n}\right)+O\left(\alpha^{n}\right)=\dot{O}\left(\alpha^{n}\right)$.

This completes the induction and shows that (17) holds. Now, (16) and (17) imply

$$
\left\|z_{\alpha, n}-\mathrm{K}^{\dagger}\left(g-\mathrm{K} L^{\dagger} f\right)\right\|=O\left(\alpha^{n}\right)
$$

so that by combining (14) and (20) we obtain the following convergence rate for our algorithm for the case of exact data: 
TheOREM 1. Let $K: H_{1} \rightarrow H_{2}$ and $L: H_{1} \rightarrow H_{3}$ be bounded linear operators and assume that $(K, L)$ has a trivial nullspace and closed range. Let $\mathrm{K}$ be defined as in Section 1 and assume (with an $n \in N$ ) that

$$
\begin{gathered}
f \in D\left(L^{\dagger}\right) \\
L^{\dagger} f \in R\left(\left(L^{\star} L\right)^{n}\right) \\
\mathrm{K}^{\dagger}\left(g-\mathrm{K} L^{\dagger} f\right) \in R\left(\left(\mathrm{~K}^{\star} \mathrm{K}\right)^{n}\right) .
\end{gathered}
$$

Then for $x_{\alpha, n}$ defined by the algorithm of Section 1 we have

$$
\left\|x_{\alpha, n}-\hat{x}\right\|=O\left(\alpha^{n}\right)
$$

where $\hat{x}$ is defined by (3) and (4).

Thus, our algorithm is faster than the method of weighting, which, in the noise-free case yields the convergence rate $O(\alpha)$ (see [2], [11]). Note that the complexity of our algorithm is not much higher than that of the method of weighting: As a comparison with $[\mathbf{1 1},(8)]$ shows, both methods involve the inversion of two linear operators. We will discuss the conditions (21) $-(23)$ at the end of this Section.

We now turn to the case of noisy data and assume that instead of $f$ and $g$, we use $f_{6}$ and $g_{\delta}$ with $\left\|f-f_{\delta}\right\| \leqslant \delta,\left\|g-g_{\delta}\right\| \leqslant \delta$ in our algorithm. The approximations resulting from (9), (11) and (12) will be denoted by $y_{\alpha, j}^{\delta}, z_{\alpha, j}^{\delta}$ and $x_{\alpha, n}^{\delta}$, respectively.

The effect of the perturbation in $f$ on $y_{\alpha, n}$ can be seen from (15). Now, for $j \in\{1, \ldots, n\}, z_{\alpha, j}^{\delta}-z_{\alpha, j}=\left(\mathrm{K}^{\star} \mathrm{K}+\alpha I\right)^{-1} \mathrm{~K}^{\star}\left(g_{\delta}-g\right)+\alpha\left(\mathrm{K}^{\star} \mathrm{K}+\alpha I\right)^{-1}\left(z_{\alpha, j-1}^{\delta}-\right.$ $\left.z_{\alpha, j-1}\right)-\left(\mathrm{K}^{\star} \mathrm{K}+\alpha I\right)^{-1} \mathrm{~K}^{\star} \mathrm{K}\left(y_{\alpha, n}^{\delta}-y_{\alpha, n}\right)$. From this formula, it follows as above (by induction) together with (15), (18), (19) and the estimates $\left\|g_{\delta}-g\right\| \leqslant \delta$ and $\left\|\left(\mathrm{K}^{\star} \mathrm{K}+\alpha I\right)^{-1} \mathrm{~K}^{\star}\right\|=O\left(\frac{1}{\sqrt{\alpha}}\right)$ that

$$
\left\|z_{\alpha, n}^{\delta}-z_{\alpha, n}\right\|=O\left(\frac{\delta}{\sqrt{\alpha}}\right) .
$$

Now, (15) and (25) imply together with the result from Theorem 1 that under its assumptions,

$$
\left\|x_{\alpha, n}^{\delta}-\hat{x}\right\|=O\left(\alpha^{n}\right)+O\left(\frac{\delta}{\sqrt{\alpha}}\right),
$$

and hence with the choice $\alpha \sim \delta^{\frac{2}{1+2 n}}$ we obtain the rate $\left\|x_{\alpha, n}^{\delta}-\hat{x}\right\|=O\left(\delta^{\frac{2 n}{1+2 n}}\right)$, which (for $n>1$ ) is better than the best possible rate $O\left(\delta^{\frac{2}{3}}\right)$ for the method of weighting. Also, for $n$ sufficiently large, this rate can be arbitarily close to the optimal rate $O(\delta)$. Of course, increasing $n$ only makes sense as long as the conditions (22) and (23) are fulfilled. Otherwise, the rate of convergence does not improve.

We summarise this discussion: 
THEOREM 2. Let the assumptions of Theorem 1 be fulfilled. Let $x_{\alpha, n}^{\delta}$ be defined by the algorithm of Section 1 with $f$ and $g$ replaced by $f_{\delta}$ and $g_{\delta}$ and assume that $\left\|f-f_{\delta}\right\| \leqslant \delta,\left\|g-g_{\delta}\right\| \leqslant \delta$.

If $\alpha=\alpha(\delta) \sim \delta^{\frac{2}{2 n+1}}$, then

$$
\left\|x_{\alpha, n}^{\delta}-\hat{x}\right\|=O\left(\delta^{\frac{2 n}{1+2 n}}\right)
$$

where $\hat{x}$ is defined by (3) and (4).

Finally, we want to discuss conditions (21)-(23): If (21) is not fulfilled, then $\lim _{\alpha \rightarrow 0}\left\|y_{\alpha, n}\right\|=+\infty$ (see [10,Th.2.1.2]). Also, the splittings (7) and (8) would not be possible, although $\hat{x}$ (and also the method of weighting) are defined (see [11]). Thus, (21) seems to be necessary for our method to work. But note that in most cases of interest (see [2]), $R(L)$ is closed anyway, in which case $D\left(L^{\dagger}\right)=H_{3}$, so that (21) is always fulfilled.

If $R(L)$ or $R(K)$, respectively, are non-closed, then the conditions (22) or (23) are actually necessary for the convergence rates (14) and (16) (see [10, Theorem 3.2.1] for $n=1$ and the compact case). Thus, these conditions cannot be relaxed. Since (23) involves the adjoint and generalised inverse with respect to the inner product (6), we interpret this condition in terms of the original structure on $H_{1}$ : First note that because of $(7),(23)$ is equivalent to

$$
\hat{\boldsymbol{x}}-L^{\dagger} f \in R\left(\left(\mathrm{~K}^{\star} \mathrm{K}\right)^{n}\right) .
$$

The range of $\left(K^{\star} K\right)^{n}$ is described as follows:

Proposition. $x \in R\left(\left(\mathrm{~K}^{\star} \mathrm{K}\right)^{n}\right)$ if and only if there exists vectors $x_{1}, \ldots, x_{n}$ with $L^{\star} L x_{1} \in R\left(K^{\star} K\right), L^{\star} L x_{j}=K^{\star} K x_{j-1}$ for $j \in\{2, \ldots, n\}, x_{n}=x$.

Proof: $x \in R\left(\left(\mathrm{~K}^{\star} \mathrm{K}\right)^{n+1}\right)$ if and only if $x \in R\left(\left(\mathrm{~K}^{\star} \mathrm{K}\right)^{n}\right)$ and then there is a $z \in R\left(\left(\mathrm{~K}^{\star} \mathrm{K}\right)^{n}\right)$ with $x=\mathrm{K}^{\star} \mathrm{K} z$. Since $\mathrm{K}^{\star}=\left(K^{\star} K+L^{\star} L\right)^{-1} K^{\star}$, this is equivalent to $L^{\star} L x=K^{\star} K(z-x)$. Hence $x \in R\left(\left(\mathrm{~K}^{\star} \mathrm{K}\right)^{n+1}\right)$ if and only if there exists a $v \in R\left(\left(\mathrm{~K}^{\star} \mathrm{K}\right)^{n}\right)$ with $L^{\star} L x=K^{\star} K v$. From this, we can prove the assertion by induction.

This proposition together with (27) gives at least some feeling for condition (23).

Finally, we note the following: If $T$ is a selfadjoint bounded linear operator bet ween Hilbert spaces with closed range, then $R\left(T^{n}\right)=R(T)$ and $N\left(T^{n}\right)=N(T)$ for all $n \in N$. Hence, if $R\left(L^{\star} L\right)$ is closed, then $L^{\dagger} f \in N(L)^{\perp}=N\left(L^{\star} L\right)^{\perp}=R\left(L^{\star} L\right)=$ $R\left(\left(L^{\star} L\right)^{n}\right)$ for all $n \in \mathrm{N}$, so that in this case, (22) is fulfilled for all $n \in \mathrm{N}$. Thus, in this case, (23) is the only "real" condition that restricts the choice of $n$. 


\section{The Case of unbounded $L$}

We now indicate how the results of Section 2 extend to the case of a densely defined, closed, but not necessarily bounded linear operator $L: D(L) \subseteq H_{1} \rightarrow H_{3}$. First, we must extend the basic theory of iterated Tikhonov regularisation for approximating $L^{\dagger} f$ to that case. Note that since $f$ need not be in $D\left(L^{\star}\right),(9)$ does not have an immediate meaning. We assign a meaning to (9) via a weak formulation: One shows as in [16] that for any $\alpha>0, D(L)$ equipped with the inner product

$$
(x, y)_{\alpha}:=\langle L x, L y\rangle+\alpha\langle x, y\rangle
$$

is a Hilbert space. We consider the linear functional

$$
v \rightarrow\langle f, L v\rangle
$$

on this Hilbert space. If a sequence $\left(v_{k}\right)$ converges to $v$ with respect to the norm induced by $(\cdot, \cdot)_{\alpha}$, then, since $L$ is continuous with respect to this norm, $\left(L v_{k}\right)$ converges to $L v$ and hence $\left(\left\langle f, L v_{k}\right\rangle\right)$ converges to $\langle f, L v\rangle$. Hence, the linear functional in (29) is bounded on $\left(D(L),(\cdot, \cdot)_{\alpha}\right)$. Thus, by the Riesz Representation Theorem, there is a unique $y_{\alpha, 1} \in D(L)$ such that

$$
\left\langle L y_{\alpha, 1}, L v\right\rangle+\alpha\left\langle y_{\alpha, 1}, v\right\rangle=\left(y_{\alpha, 1}, v\right)_{\alpha}=\langle f, L v\rangle
$$

for all $v \in D(L)$. This is the weak formulation of Tikhonov regularisation. Moreover, if $f \in D\left(L^{\dagger}\right)$ and $y=L^{\dagger} f$, then $\langle f, L v\rangle=\langle L y, L v\rangle$ for all $v \in D(L)$ (which is the weak formulation of the normal equations) and hence (because of (30))

$$
\alpha\left\langle y_{\alpha, 1}-y, v\right\rangle+\left\langle L\left(y_{\alpha, 1}-y\right), L v\right\rangle=-\alpha\langle y, v\rangle
$$

for all $v \in D(L)$. Now, (31) implies that $y_{\alpha, 1}-y \in D\left(L^{\star} L\right)$ and that

$$
\left(\alpha I+L^{\star} L\right)\left(y_{\alpha, 1}-y\right)=-\alpha y
$$

holds. Because of von Neumann's Lemma (see [12]), $\left(\alpha I+L^{\star} L\right)^{-1}$ is bounded and $\left\|\left(\alpha I+L^{\star} L\right)^{-1}\right\| \leqslant \frac{1}{\alpha}$. Thus, (32) can be written in the form

$$
y_{\alpha, 1}-y=-\alpha\left(\alpha I+L^{\star} L\right)^{-1} y
$$

From (33), convergence rates can be derived. For example, if $y \in R\left(L^{\star} L\right)$, then with $L^{\star} L z=y$ we obtain $y_{\alpha, 1}-y=-\alpha\left(\alpha I+L^{\star} L\right)^{-1} L^{\star} L z=-\alpha z+\alpha^{2}\left(\alpha I+L^{\star} L\right)^{-1} z$ and hence $\left\|y_{\alpha, 1}-y\right\|=O(\alpha)$. Analogously, we can define iterated Tikhonov regularisation 
via this weak formulation: Let $y_{\alpha, 0}=0$ and let, for $j \in\{1, \ldots, n\}, y_{\alpha, j}$ be the unique element in $D(L)$ satisfying

$$
\left\langle L y_{\alpha, j}, L v\right\rangle+\alpha\left\langle y_{\alpha, j}, v\right\rangle=\langle f, L v\rangle+\alpha\left\langle y_{\alpha, j-1}, v\right\rangle
$$

for all $v \in D(L)$. It follows as above that $y_{\alpha, j}-y \in D\left(L^{\star} L\right)$ and that

$$
y_{\alpha, j}-y=-\alpha\left(\alpha I+L^{\star} L\right)^{-1}\left(y-y_{\alpha, j-1}\right)
$$

holds for all $j \in\{1, \ldots, n\}$. Hence we can prove as above that if $y \in R\left(\left(L^{\star} L\right)^{n}\right)$, then $\left\|y_{\alpha, n}-y\right\|=O\left(\alpha^{n}\right)$.

Now assume that $\left\|f-f_{\delta}\right\| \leqslant \delta$ and define $y_{\alpha, n}^{\delta}$ by (34) with $f$ replaced by $f_{\delta}$. Then

$$
\left\langle L\left(y_{\alpha, j}-y_{\alpha, j}^{\delta}\right), L v\right\rangle+\alpha\left\langle y_{\alpha, j}-y_{\alpha, j}^{\delta}, v\right\rangle=\left\langle f-f_{\delta}, L v\right\rangle+\alpha\left\langle y_{\alpha, j-1}-y_{\alpha, j-1}^{\delta}, v\right\rangle
$$

for all $v \in D(L)$ and $j \in\{1, \ldots, n\}$. We claim that for all $j \in\{1, \ldots, n\}$,

$$
\left\|y_{\alpha, j}-y_{\alpha, j}^{\delta}\right\|=O\left(\frac{\delta}{\sqrt{\alpha}}\right)
$$

holds. For $j=1$ we obtain from (36) with $v:=y_{\alpha, 1}-y_{\alpha, 1}^{\delta}$,

$$
\left\|L\left(y_{\alpha, 1}-y_{\alpha, 1}^{\delta}\right)\right\|^{2}+\alpha\left\|y_{\alpha, 1}-y_{\alpha, 1}^{\delta}\right\|^{2}=\left\langle f-f_{\delta}, L\left(y_{\alpha, 1}-y_{\alpha, 1}^{\delta}\right)\right\rangle \leqslant \delta\left\|L\left(y_{\alpha, 1}-y_{\alpha, 1}^{\delta}\right)\right\|
$$

From this, we first obtain $\left\|L\left(y_{\alpha, 1}-y_{\alpha, 1}^{\delta}\right)\right\| \leqslant \delta$ and then $\alpha\left\|y_{\alpha, 1}-y_{\alpha, 1}^{\delta}\right\|^{2} \leqslant \delta^{2}$, that is, (37) with $j=1$. Now we can proceed by induction: Assume that (37) holds for $j-1$ and use (36) with $v:=y_{\alpha, j}-y_{\alpha, j}^{\delta}$. Then we obtain (with the abbreviations $a:=$ $\left.\left\|L\left(y_{\alpha, j}-y_{\alpha, j}^{\delta}\right)\right\|, b:=\left\|y_{\alpha, j}-y_{\alpha, j}^{\delta}\right\|\right): a^{2}+\alpha b^{2} \leqslant \delta a+\alpha \frac{c \delta}{\sqrt{\alpha}} b$ with a suitable constant $c>0$. The right-hand side of this inequality is dominated by $\frac{\delta^{2}}{2}+\frac{a^{2}}{2}+\frac{c^{2} \delta^{2}}{2}+\frac{\alpha b^{2}}{2}$. We thus obtain $\frac{a^{2}}{2}+\frac{\alpha b^{2}}{2} \leqslant \delta^{2} \cdot\left(\frac{1}{2}+\frac{c^{2}}{2}\right)$. This now implies that $b=O\left(\frac{\delta}{\sqrt{\alpha}}\right)$. By definition of $b$, this is just (37) for $j$, which completes the induction. Thus (37) is verified.

We summarise these results:

Theorem 3. Let $L$ be a densely defined closed linear operator between Hilbert spaces, $f \in D\left(L^{\dagger}\right)$ and assume that (22) holds for an $n \in \mathrm{N}$. Let $y_{\alpha, n}$ be defined by (36). Then

$$
\left\|y_{\alpha, n}-L^{\dagger} f\right\|=O\left(\alpha^{n}\right)
$$

holds. If $\left\|f-f_{\delta}\right\| \leqslant \delta$ and $y_{\alpha, n}^{\delta}$ is defined by (34) with $f$ replaced by $f_{\delta}$, then

$$
\left\|y_{\alpha, n}-y_{\alpha, n}^{\delta}\right\|=O\left(\frac{\delta}{\sqrt{\alpha}}\right)
$$


holds. Hence, if $\alpha=\alpha(\delta) \sim \delta \frac{2}{1+2 n}$, then

$$
\left\|y_{\alpha, n}^{\delta}-L^{\dagger} f\right\|=O\left(\delta^{\frac{2 n}{1+2 n}}\right) .
$$

This theorem extends the theory of iterated Tikhonov regularisation developed in $[13]$ to the case of an unbounded operator $L$. Now, we come back to our algorithm of Section 1 for approximating the restricted least-squares solution $\hat{x}$. The fact that $L$ is now unbounded affects only the $y_{\alpha, n}^{\delta}$. The other part of the iteration, namely $z_{\alpha, n}^{\delta}$, can be handled as in Section 2. This implies:

THEOREM 4. The statements of Theorems 1 and 2 hold if (with all other assumptions unchanged) $L$ is closed and densely defined, if $\left(y_{\alpha, n}\right)$ and $\left(y_{\alpha, n}^{\delta}\right)$ are defined by (34) (with $f$ and $f_{\delta}$ ) instead of (9), the rest of the algorithm being unchanged.

The following extensions should be possible (both for our algorithm and for the weak form of iterated Tikhonov regularisation per se):

(1) A posteriori parameter choice rules like the ones in [6], [9].

(2) Superconvergence results as in[19], and a posteriori parameter choice rules for getting superconvergence with optimal rates $([8])$. This is relevant for the "linear functional strategy" for solving ill-posed problems (see [1]).

(3) Finite element realisations of our algorithm like the one given for the method of weighting in [3].

(4) Projection methods for approximating $\hat{x}$ in the spirit of [7].

We do not elaborate on these points.

\section{REFERENCES}

[1] R.S. Anderssen, 'The linear functional strategy for improperly posed problems', in Inverse Problems, J.Cannon, U. Hornung (eds.), pp. 11-30 (Birkhäuser, Basel, 1986).

[2] G.D.Callon, 'Theory and approximation of the restricted pseudosolution', Ph.D thesis, Univ. of Cincinnati.

[3] G.D.Callon, 'Finite element approximations for restricted pseudosolutions' (to appear).

[4] G. D. Gallon and C.W. Groetsch, 'The method of weighting and approximation of restricted pseudosolutions', J. Approx. Theory 51 (1987), 11-18.

[5] H.W. Engl, 'Methods for approximating solutions of ill-posed linear operator equations based on numerical functional analysis', in Methods of Functional Analysis in Approximation Theory, C.A. Micchelli, D.V. Pai and B.V. Limaye (eds.), pp. 337-355 (Birkhäuser, Basel, 1986).

[6] H.W. Engl, 'On the choice of the regularization parameter for iterated Tikhonov regularization of ill-posed problems', J. Approx. Theory 49 (1987), 55-63.

[7] H.W. Engl and A. Neubauer, 'On projection methods for solving linear ill-posed problems' (to appear), in Model Optimization in Exploration Geophysics, Edited by A. Vogel (Vieweg, Wiesbaden, 1987 to appear).

[8] H.W. Engl and A. Neubauer, 'A parameter choice strategy for (iterated) Tikhonov regularization of ill-posed problems leading to superconvergence with optimal rates', Applic. Analysis (to appear). 
[9] H. Gfrerer, 'An a-posteriori parameter choice for ordinary and iterated Tikhonov regularization of ill-posed problems leading to optimal convergence rates', Math. Comp. (to appear).

[10] C.W. Groetsch, The Theory of Tikhonov Regularization for Fredholm Equations of the First Kind (Pitman, Boston, 1984).

[11] C.W. Groetsch, 'Regularization with equality constraints', in Inverse Problems: Lecture Notes in Math. 1225, G. Talenti (ed.), pp. 169-181 (Springer, New York, 1986).

[12] T. Kato, Perturbation Theory for Linear Operators, $2^{\text {nd }}$ ed. (Springer, Berlin, 1976).

[13] J.T. King and D. Chillingworth, 'Approximation of generalized inverses by iterated regularization', Numer. Funct. Anal. Optim. 1 (1979), 499-513.

[14] S.J. Lee, 'Tikhonov's regularization of an arbitrary closed linear operator', Abstracts of papers presented to the Amer. Math. Soc. 8 (1987), p. 82.

[15] S.J. Lee and M.Z. Nashed, 'Constrained minimization problems for linear relations in Hilbert spaces' (to appear).

[16] J. Locker and P. Prenter, 'Regularization with differential operators I: General theory', J. Math. Anal. Appl. 74 (1980), 504-529.

[17] A. Morozov, Methods for Solving Incorrectly Posed Problems (Springer, New York, 1984).

[18] M. Z. Nashed, 'Aspects of generalized inverses in analysis and regularization', in Generalized Inverses and Applications, M. Z. Nashed (ed.), pp. 193-244 (Academic Press, New York, 1976).

[19] E. Schock, 'Approximate solutions to ill-posed problems: arbitrarily slow convergence vs. superconvergence', in Constructive Methods for the Practical Treatment of Integral Equations, G. Hämmerlin and K.H. Hoffmann (eds.), pp. 234-243 (Birkhäuser, Basel, 1985).

[20] M.R. Trummer, 'A method of solving ill-posed linear operator equations', SIA M J. Numer. Anal. 21 (1984), 729-737.

Professor H. Engl, Institut für Mathematik, Universität, A-9022 KLAGENFURT, Austria.

Professor H. Engl, Institut für Mathematik Johannes-Kepler-Universität

A-4040 LINZ

Austria
Professor C.W. Groetsch, Department of Mathematical Sciences, University of Cincinnati, CINCINNATI, OH. 45221-0025 U.S.A. 